\title{
Determining standard criteria for tubeless PCNL
}

\author{
Mansur Daggülli · Haluk Söylemez • \\ Mehmet Nuri Bodakci • Namık Kemal Hatipoglu
}

Received: 13 February 2014 / Accepted: 22 February 2014 / Published online: 11 March 2014

(C) Springer-Verlag Berlin Heidelberg 2014

Dear Sir,

We read the article by Rifaioglu et al. [1] with great interest. This study makes a valuable contribution to the literature by elucidating determination of predictive factors for the placement of a percutaneous nephrostomy tube (PNT) during a percutaneous nephrolithotomy (PCNL) procedure. The authors also aimed to evaluate the optimal cut-off points for the predictive factors. However, we have some concerns on the methods of the study.

First of all, this is a retrospectively designed multicenter study, and as the authors stated, the decision to place a PNT at the end of the procedure depended on the surgeon's discretion. Their indications for tubeless PCNL were minimal bleeding during the case, no evidence of collecting system perforation, and low index of suspicion for residual stone fragments. All these indications depend on subjective criteria, which vary from one surgeon to another. They then compared the tubeless PCNL group with the standard PCNL group according to the incidence of perioperative complications, estimated blood loss, length of hospital stay, and treatment success. This comparison is not suitable for such studies because tubeless PCNL was performed in selected cases; hence, comparisons based on these parameters would not be meaningful. For instance, if a surgeon placed a PNT in patients whose bleeding during the case was high, then their hemoglobin level would be lower than patients in the tubeless group. So, we cannot make a decision to perform tubeless PCNL in a patient in light of these results. We suggest that a randomized, prospective, singlecenter study should be done to determine the predictive factors for placement of a PNT.

Finally, the authors need to state whether they placed a ureteral catheter (totally tubeless PCNL). If so, they need to provide the data regarding its removal.

Conflict of interest None.

\section{References}

1. Rifaioglu MM, Onem K, Buldu I, Karatag T, Istanbulluoglu MO (2014) Tubeless percutaneous nephrolithotomy: yes but when? A multicentre retrospective cohort study. Urolithiasis. doi:10.1007/ s00240-014-0638-3. (Epub ahead of print)

\footnotetext{
M. Daggülli $(\bowtie) \cdot$ H. Söylemez · M. N. Bodakci ·

N. K. Hatipoglu

Department of Urology, Medical Faculty,

Dicle University, Diyarbakir, Turkey

e-mail: mansurdaggulu@yahoo.com

H. Söylemez

e-mail: drhaluks@yahoo.com
}

M. N. Bodakci

e-mail: mehmetbodakci@yahoo.com

N. K. Hatipoglu

e-mail: n.hatipoglu52@mynet.com 